This is a provisional PDF only. Copyedited and fully formatted version will be made available soon.

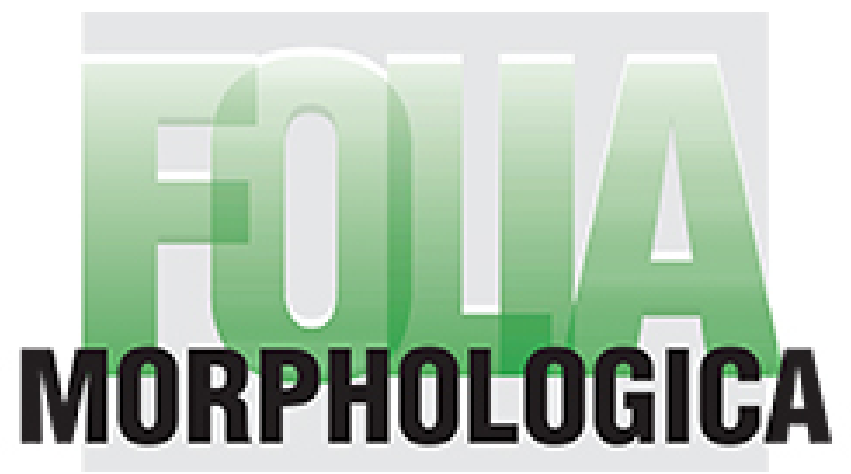

ISSN: 0015-5659

\title{
A unilateral sternopharyngeal branch of the sternocleidomastoid muscle in an aged Caucasian male: a unique cadaveric report
}

Authors: S. Silawal, S. Morgan, L. Ruecker, G. Schulze-Tanzil

DOI: $10.5603 / F M . a 2022.0016$

Article type: Case report

Submitted: $2021-12-06$

Accepted: $2022-01-12$

Published online: 2022-02-17

This article has been peer reviewed and published immediately upon acceptance.

It is an open access article, which means that it can be downloaded, printed, and distributed freely, provided the work is properly cited.

Articles in "Folia Morphologica" are listed in PubMed. 


\section{A unilateral sternopharyngeal branch of the sternocleidomastoid muscle in an aged Caucasian male: a unique cadaveric report}

S. Silawal et al., A sternopharyngeal muscular branch variation in a cadaveric study

S. Silawal, S. Morgan, L. Ruecker, G. Schulze-Tanzil

Institute of Anatomy and Cell Biology, Paracelsus Medical University, Nuremberg and Salzburg, General Hospital Nuremberg, Nuremberg, Germany

Address for correspondence: Dr. med. Sandeep Silawal, Institute of Anatomy and Cell Biology, Paracelsus Medical University, Salzburg and Nuremberg, Prof. Ernst Nathan Str. 1, 90419 Nuremberg, Germany, tel:+49 - (0)911-398-116771, fax: +49 - (0)911-398-6774, email: sandeep.silawal@pmu.ac.at; sandeep.silawal@klinikum-nuernberg.de

\footnotetext{
Abstract

The sternocleidomastoid muscle (SCM) consists of a sternal and a clavicular head which merge together and inserts distally posterolateral on the mastoid process and superior nuchal line, hence separating the anterior from the posterior triangle of the neck. Many types of structural variations in SCM have already been reported before. A unique variation of this muscle was discovered in an aged caucasian male cadaver during an anatomical dissection at the Paracelsus Medical University in Nuremberg, Germany.

This study reports a right unilateral accessory muscular branch at the sternal head of the SCM which formed a tendon on the level of omohyoid muscle before dividing into anterior and posterior fascicles. The posterior fascicle attached to the external carotid artery (ECA) at the site where a common trunk for lingual and facial artery (cLA/FA) branched off, drawing ECA inferiorly to build an inferior loop, whereas the anterior fascicle passed further superior and broadened to form a muscular belly. This superior muscular belly extended to the posterior and lateral side of the pharynx to ultimately merge into the superior constrictor pharyngeal muscle. Such anatomical variation has never been reported before.

Therefore, we propose the nomenclature of this variational structure as a sternopharyngeal branch of the sternocleiodomastoid muscle. This report helps not only to inform the clinicians
} 
regarding the possible variation of this muscle during surgical procedures or radiological diagnostics but also encourage developmental researches in the future.

Key words: sternopharyngeal, sternocleidomastoid, sternocervical, variation

\section{INTRODUCTION}

The sternocleidomastoid muscle (SCM) borders anteriorly the posterior triangle of the neck and limits the anterior triangle of the neck posteriorly. As per the anatomical literature, this muscle consists of a sternal and a clavicular head which merge together and attaches posterolateral to the mastoid process and the superior nuchal line. Structures like spinal accessory nerve, branches of the cervical plexus, roots and trunks of the brachial plexus, phrenic nerve, subclavian artery, transverse cervical artery, external jugular vein, inferior belly of the omohyoid, scalene muscles, splenius muscle, levator scapulae muscle, occipital supraclavicular lymph nodes etc. lie in a close proximity posterior to the SCM. Submental, submandibular, superior and inferior carotid triangles are the regions in the anterior triangle of the neck. Common carotid artery, branches of the external carotid artery (ECA), the internal jugular vein, vagus nerve, branches of the ansa cervicalis are some of the examples of vital structures that are located in this triangle anterior to the SCM (1). Anatomical variations of SCM could impact on above neighboring structures via compression or narrowing of the surrounding space. Also, understanding the relevance of such variations is very important during surgical interventions in the neck area such as neck dissection. Various anomalies in the proximal heads of SCM have been described before (2-17). During the developmental process of the shoulder girdle musculature, SCM and trapezoid muscles derive from the lateral plate mesoderm as a common embryonic source $(18,19)$. The splitting of the embryological common trapezius-sternocleidomastoideus muscle system leads to two individual muscles of the neck, where they still share the common innervation of the accessory nerve (20). This process of separation could result in different forms of muscle variations. Even though the variations in the proximal head are reportedly commonly, the variations described in the insertional end are not that common (21, 22). A particular muscular variation, the so called cleidocervical or levator claviculae muscle shows that the muscle is attached proximally to the clavicle and distally to different levels of the cervical vertebra (23). Our study reports a special variation of the SCM where a muscular branch from the sternal side finds non-osseous attachment sites which makes the case report unique. 


\section{CASE REPORT}

During an anatomical dissection class at the Paracelsus Medical University, we discovered a $0.3 \mathrm{~cm}$ thick (maximum thickness) accessory muscular branch of the sternal head of the right SCM in a formalin-fixed 102-year-old caucasian male (Figure 1A). The cadavers are registered in the Munich body donation program and contains written consents of the body donors. This inferior muscular belly deviated medially towards the carotid triangle. $6.5 \mathrm{~cm}$ distal from the sternal end, on the level of the crossing of the omohyoid muscle, the tendon of this muscle was divided into anterior and posterior fascicles (Figures 1A-C). The posterior fascicle was attached to the ECA (Figure 1B-C). The insertion of this fascicle was located at a site where a common trunk for lingual and facial artery (cLA/FA) was branched from the ECA (Figure 1B-C). Grade I kinking of the ICA was observed (Figure 1B-C) (24). The anterior fascicle passed further superior in front of cLA/FA before it broadened to form a superior muscular belly (Figure 1B-D) (\#). This muscular belly then passed towards the posterior and lateral side of the pharynx to ultimately merge into the superior constrictor pharyngeal muscle (Figure 1D). Glossopharyngeal nerve pierced the superior muscular belly at the narrow proximal section before it made a loop towards anterior (Figure 1D). In comparison, the corresponding left side of the neck was void of this sternopharyngeal muscular variation (Figure 2).

\section{DISCUSSION}

A small morphological study in an indian population with 18 cadavers showed that $27.8 \%$ of the specimens had SCM variations with three unilateral and two bilateral presentations (6). A different study describes a strap-like muscle originating from the middle third of the clavicle inserting at the transverse process of the C3 vertebra (14). The level of cervical attachments of these cleidocervical (levator claviculae) muscles can however, vary from atlas to the $6^{\text {th }}$ cervical vertebra as reported in a literature survey by Obate et al on 2012 (23). In another study with CT scans, this muscle was identified in 6 subjects out of 300 subjects, which makes the prevalence of this muscle of about $2 \%$ (25). A unique variation has been reported in our study. An accessory muscular branch from the sternal head split into anterior and posterior fascicles. Since the posterior fascicle of this muscular branch was attached to ECA, a muscular contraction during any movement of the head could have possible traction to this artery inferiorly. Any symptomatic consequence to this arterial traction cannot be followed back, however, the inferior looping of ECA was detected which could be a possible anatomical result of continuous traction during the development. The 
anterior fascicle ran in front of cLA/FA and built another thin muscular belly. This muscle ran upwards towards the posterior and lateral side of the pharynx and merged into the right superior pharyngeal constrictor muscle. Since the glossopharyngeal nerve penetrated this superior belly of the sternopharyngeal muscular branch, we assume that this nerve should be responsible for the innervation of the superior muscular belly. However, the inferior belly would be rather distant from the glossopharyngeal nerve, hence it would be more appropriate to be innervated by the accessory nerve. Unfortunately, we cannot prove this hypothesis in this case report. Finally, we propose a proper nomenclature for this variation as a sternopharyngeal branch of the SCM. Even though two fascicles of this muscular branch were observed, we assume that they resulted from splitting of a single muscle during the embryological development. The muscular fibers close to the ECA could have mal-attached to the artery. The pulsating behavior of the artery could have split the muscle away from its original position. This hypothesis however has not been proven yet and would obviously need further investigation.

\section{CONCLUSIONS}

This manuscript reports a unique case of right sided unilateral muscular variation derived from the sternal head of the SCM ultimately merging into the right superior constrictor pharyngeal muscle. Such anatomical variation has never been reported before. Therefore, we propose the nomenclature of this variational structure as a sternopharyngeal branch of the sternocleiodomastoid muscle. This novel discovery broadens the spectrum of SCM variations and help surgeons or physicians to understand the possible anatomy of neck while accessing the anterior triangle of the neck for any clinical purposes.

\section{Acknowledgements}

We are very thankful to the body donors for their contribution to the academic teaching and research field of anatomy.

Conflict of interest: None declared

\section{REFERENCES}

1. Kohan EJ, Wirth GA. Anatomy of the neck. Clinics in plastic surgery. 2014;41(1):1-6. Epub 2013/12/04. doi: 10.1016/j.cps.2013.09.016. PubMed PMID: 24295343.

2. Heo YR, Kim JW, Lee JH. Variation of the sternocleidomastoid muscle: a case report of three heads and an accessory head. Surgical and radiologic anatomy : SRA. 2020;42(6):711-3. Epub 2019/11/27. doi: 10.1007/s00276-019-02388-4. PubMed PMID: 31768700. 
3. Kim S-Y, Jang H-B, Kim J, Yoon SP. Bilateral four heads of the sternocleidomastoid muscle. Surgical and Radiologic Anatomy. 2015;37(7):871-3. doi: 10.1007/s00276-014-1397-0.

4. Ramesh Rao T, Vishnumaya G, Prakashchandra S, K, Suresh R. Variation in the Origin of Sternocleidomastoid Muscle: A Case Report. International Journal of Morphology. 2007;25:621-3.

5. Mansoor SN, Rathore FA. Accessory Clavicular Sternocleidomastoid Causing Torticollis in an Adult. Prog Rehabil Med. 2018;3:20180006-. doi: 10.2490/prm.20180006. PubMed PMID: 32789231.

6. Saha A, Mandal S, Chakraborty S, Bandyopadhyay M. Morphological study of the attachment of sternocleidomastoid muscle. Singapore Med J. 2014;55(1):45-7. doi: 10.11622/smedj.2013215. PubMed PMID: 24241357.

7. Oh JS, Kim CE, Kim J, Yoon SP. Bilateral supernumerary clavicular heads of sternocleidomastoid muscle in a Korean female cadaver. Surgical and radiologic anatomy : SRA. 2019;41(6):699-702. Epub 2019/03/29. doi: 10.1007/s00276-019-02227-6. PubMed PMID: 30919044.

8. Natsis K, Asouchidou I, Vasileiou M, Papathanasiou E, Noussios G, Paraskevas G. A rare case of bilateral supernumerary heads of sternocleidomastoid muscle and its clinical impact. Folia morphologica. 2009;68(1):52-4. Epub 2009/04/23. PubMed PMID: 19384831.

9. Cherian SB, Nayak S. A Rare Case of Unilateral Third Head of Sternocleidomastoid Muscle. International Journal of Morphology. 2008;26:99-101.

10. Nayak SR, Krishnamurthy A, Kumar Sj M, Pai MM, Prabhu LV, Jetti R. A rare case of bilateral sternocleidomastoid muscle variation. Morphologie. 2006;90(291):203-4. doi: https://doi.org/10.1016/S1286-0115(06)74507-6.

11. Amorim Júnior AAd, Lins CCdSA, Cardoso APdS, Damascena CG. Variation in Clavicular Origin of Sternocleidomastoid Muscle. International Journal of Morphology. 2010;28:97-8.

12. Kaur D, Jain M, Shukla L. Six heads of origin of sternocleidomastoid muscle: a rare case. Internet Journal of Medical Update - EJOURNAL. 2013;8:62-4.

13. Fulmali DG, Thute PP, Keche HA, Chimurkar VK. Variant Sternocleidomastoid with Extra Clavicular Head--A Case Report. Journal of Evolution of Medical and Dental Sciences. 2020;9:3258+.

14. Raikos A, Paraskevas GK, Triaridis S, Kordali P, Psillas G, Brand-Saberi B. Bilateral Supernumerary Sternocleidomastoid Heads with Critical Narrowing of the Minor and Major Supraclavicular Fossae: Clinical and Surgical Implications. International Journal of Morphology. 2012;30:927-33.

15. Arquez HF. Muscular Variation In The Neck Region With Narrowing Of The Minor And Major Supraclavicular Fossa. International Archives of Medicine. 2017;10.

16. Rr F, Reddy A. Accessory Clavicular Head of Sternocleidomastoid Muscle: A Case Report. Sch J Med Case Rep. 2015;3(9B):865-7. PubMed PMID: article.

17. Anil A, Yaşar YK, Anil F, Coşkun ZK, Peker T. Variation of bilateral multiheaded sternocleidomastoid muscle. Gazi Medical Journal. 2017;28:56-7. doi: 10.12996/gmj.2017.17.

18. Pu Q, Huang R, Brand-Saberi B. Development of the shoulder girdle musculature. Developmental Dynamics. 2016;245(3):342-50. doi: https://doi.org/10.1002/dvdy.24378.

19. Theis S, Patel K, Valasek P, Otto A, Pu Q, Harel I, et al. The occipital lateral plate mesoderm is a novel source for vertebrate neck musculature. Development. 2010;137(17):2961-71. doi: 10.1242/dev.049726.

20. Fujita T. The Smaller Occipital Nerve, Its Topographic Relation to the Trapezius-Sternocleidomastoideus Muscle System. A Consideration on the Factors Determining the Course of a Nerve. Okajimas Folia Anatomica Japonica. 1959;33(4):217-24. doi: 10.2535/ofaj1936.33.4_217.

21. Mehta V, Arora J, Kumar A, Nayar AK, Ioh HK, Gupta V, et al. Bipartite clavicular attachment of the sternocleidomastoid muscle: a case report. Anat Cell Biol. 2012;45(1):66-9. Epub 2012/03/31. doi: 10.5115/acb.2012.45.1.66. PubMed PMID: 22536555.

22. Sarikcioglu L, Donmez BO, Ozkan O. Cleidooccipital muscle: an anomalous muscle in the neck region. Folia morphologica. 2001;60(4):347-9. Epub 2002/01/05. PubMed PMID: 11770348.

23. Odate T, Kawai M, Iio K, Funayama S, Futamata H, Takeda S. Anatomy of the levator claviculae, with an overview and a literature survey. Anatomical science international. 2012;87(4):203-11. Epub 2012/08/26. doi: 10.1007/s12565-012-0148-8. PubMed PMID: 22923186.

24. Metz H, Bannister RG, Murray-Leslie RM, Bull JWD, Marshall J. Kinking of the Internal Caroid Artery: in Relation to Cerebrovascular Disease. The Lancet. 1961;277(7174):424-6. doi: https://doi.org/10.1016/S0140-6736(61)90004-6.

25. Rubinstein D, Escott EJ, Hendrick LL. The Prevalence and CT Appearance of the Levator Claviculae Muscle: A Normal Variant Not To Be Mistaken for an Abnormality. American Journal of Neuroradiology. 1999;20(4):583-6.

Figure 1. Chronological stages of dissections of the neck $(A-D)$. The digastric sternopharyngeal muscle branch (*) of the sternocleidomastoid muscle (SCM) along the sternal head and the division into anterior and posterior tendinous fascicles. The anterior fascicle continues to form a superior muscular belly (\#). (A) Anterolateral view of the neck. 
The sternal head of the SCM has been detached and moved to the lateral side. The proximal end of the sternohyoid muscle (SHM) has been folded towards the lateral side. (B) Lateral view of the neck. The facial vein has been removed. The musculotendinous junction of the sternopharyngeal muscular branch held by forceps with a light pull inferiorly to develop strain in the two tendinous fascicles. (C) Closer view of the superior carotid triangle. The distal part of the external carotid artery (ECA) has been folded lightly towards the lateral side to show the superior muscular belly (\#). (D) Retropharyngeal view of the right lateral side. Insertion of the superior muscular belly (\#) of the sternopharyngeal muscle to form part of the superior constrictor pharyngeal muscle. The muscle pierced by the glossopharyngeal nerve (GPN). Further abbreviations used: $\mathrm{CCA}=$ Common carotid artery, $\mathrm{Cl}=$ Clavicle, $\mathrm{cLA} / \mathrm{FA}=$ Common branch of lingual and facial artery, DM = Digastric muscle, FA = Facial artery, Fm = Foramen Magnum, GH = Greater horn of the hyoid bone, HN= Hypoglossal nerve, ICA = Internal carotid artery, IJV = Internal jugular vein, $\mathrm{OHM}=$ Omohyoid muscle, $\mathrm{Ph}=$ Pharynx, $\mathrm{Pl}=$ Platysma, SPM = Stylopharyngeal muscle, $\mathrm{St}=$ Sternum, SLA/SLN = Superior laryngeal artery/nerve, STM = Sternothyroid muscle, $\mathrm{TC}=$ Thyroid cartilage, $\mathrm{TG}=$ Thyroid gland.

Figure 2. Retropharyngeal overview for the comparison of both sides. Insertion of the superior muscular belly (\#) of the sternopharyngeal muscle to form part of the superior constrictor pharyngeal muscle (red triangle). The inferior section of the muscle has been lifted and retracted to the lateral side. Abbreviations used: $\mathrm{Ph}=$ Pharynx, GPN = Glossopharyngeal nerve, $\mathrm{GH}=$ Greater horn of the hyoid bone, SPM = Stylopharyngeal muscle. 

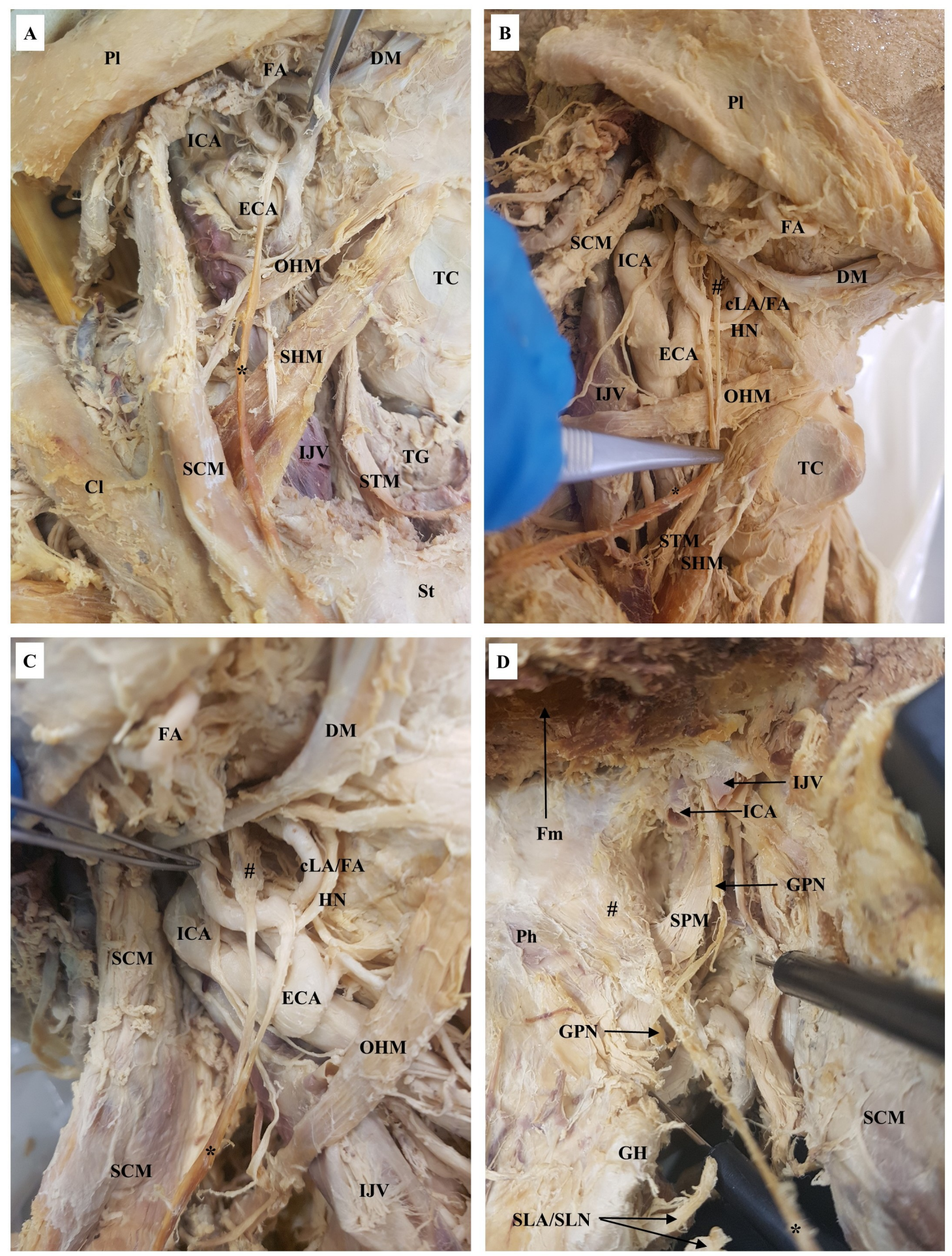


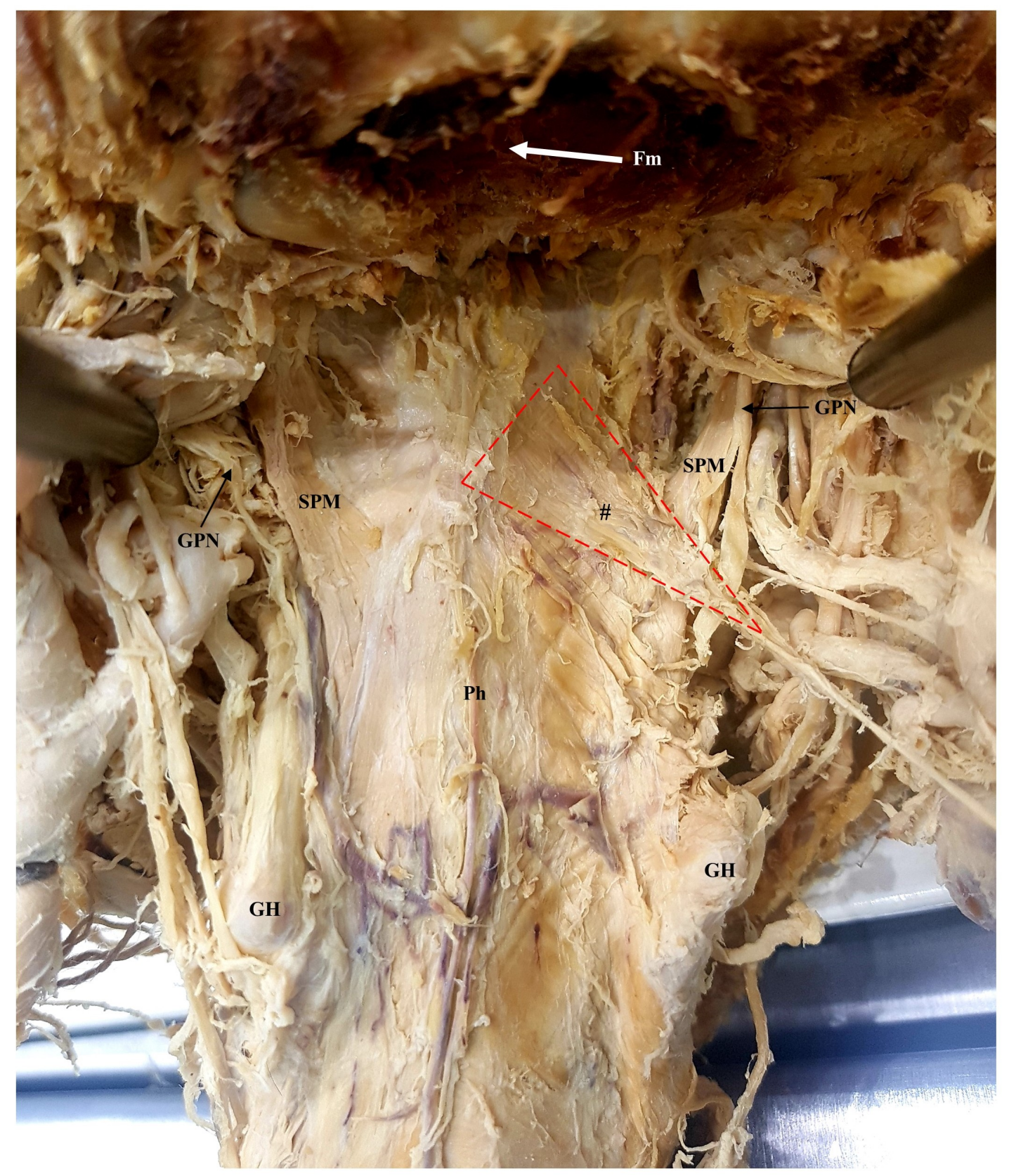

